\title{
Quantitative Annular Dark Field Images of Silicon (001) Crystal
}

\author{
Y. Oshima*, S. Kim**, Y. Tanishiro**, and K. Takayanagi** \\ * Research center for ultra HVEM, Osaka University, 7-1 Midorigaoka, Ibaraki, 567-0047, Japan \\ ** Department of Condensed Matter Physics, Tokyo Institute of Technology, 2-12-1-H-51 Oh- \\ okayama, Meguro-ku, Tokyo 152-8551, Japan
}

Three-dimensional structure (elementary) analysis of nano-scaled materials is increasingly demanded for understanding properties of electronic devices, catalysis, and rechargeable batteries. High angle annular dark-field (HAADF) imaging with scanning transmission electron microscopy (STEM) is one of powerful tools to fulfill such requirements. Recently, spherical aberration corrected electron microscopes (SAC-EM) have improved not only spatial resolution but also depth resolution as demonstrated with hafnium (Hf) atoms in silicon (Si) crystal [1]. Increased depth resolution by large convergent angle of $30 \mathrm{mrad}(300 \mathrm{keV})$ enables detection of light arsenic (As) dopants in silicon crystals [2] and of antimony clusters [3].

Recently, Stemmer et al. counted the number of electrons falling onto the annular detector and compared the absolute intensity between the experimental and simulated HAADF images for $\mathrm{SrTiO}_{3}$ and $\mathrm{PbWO}_{4}$ crystals [4-6]. They found that the absolute intensity of the experimental images matched that of the simulated images by convoluting it by a Gaussian function and suggested that the discrepancy between the experimental and simulated images came from spatial incoherence stemming from the effective size of the source, mechanical instability of the microscope, drift, and so on [4-6]. In three-dimensional analysis of atomic structure, it is important to find a factor that covers the mismatch between the absolute intensities of the experiment and simulation for the case of a large convergent angle of incident probe.

In this study [7], we performed a quantitative analysis of ADF images of non-doped silicon [001] crystals with thicknesses from 10 to $50 \mathrm{~nm}$. A multi-slice simulation based on the absorptive potential approximation was used for quantitative comparison with the experiment.

The specimens were observed by a sub-50 pm resolution STEM (R005) [8] at $300 \mathrm{keV}$. The probe converged of $30 \mathrm{mrad}$ in semi-angle. The detector inner and outer angle of 42-104 mrad was used. The ADF images were taken with a frame size of 512 × 512 pixels. The size of each pixel was 9.1 $\mathrm{pm}$. The dwell time $t$ per pixel was $38 \mathrm{us}$. The probe current was measured to be constant (30pA). Therefore, the number of incident electrons for each pixel was calculated to be $\sim 7000$ by $t^{*} \mathrm{I}_{\text {Probe }} / \boldsymbol{e}$, where $\boldsymbol{e}$ is the elementary charge. We investigated that the intensity in the ADF image is proportional to the number of electrons falling onto the detector in the range of 0 to 250 electrons and it goes to zero, when the number of electrons falling onto the detector becomes zero. It is gradually saturated above 250 electrons. The ADF intensity of each column was estimated by fitting a 2D Gaussian function to the raw data using the Levenberg-Marquardt algorithm, which is a standard method in nonlinear fitting.

$$
I=I_{P} \exp \left(-\left(x-x_{0}\right)^{2} / 2 \sigma^{2}\right) \exp \left(-\left(y-y_{0}\right)^{2} / 2 \sigma^{2}\right)+I_{B},
$$

The column intensity $I_{C}$ was defined as the sum of the Gaussian amplitude $I_{P}$ and the background $I_{B}\left(I_{C}=I_{P}+I_{B}\right)$.

Figure 1 shows mean intensity, averaging among the intensities of all pixels in the ADF image, as a function of thickness. The simulated mean intensity (solid line) had the same value as the experimental intensity (circles), which suggests that inelastic scattering cross section was quantitatively appropriate in the simulation. The first row of Fig. 2 shows the ADF images observed 
at thicknesses of 49, 37, 24 and $11 \mathrm{~m}$. The intensities are displayed as a rainbow of colors. The images in the third row are the simulations. The simulated column intensity does not match the observed one; the simulated column has a much sharper profile than the experimental one. After convolving the simulated image by a Gaussian function with an full width half maximum (FWHM) of $70 \mathrm{pm}$, the theoretical results were able to reproduce all of the experimental images from different specimen thicknesses as shown in the second row of Fig. 2.

We considered effect of lattice vibration, strain in the specimen, sample preparation and the source size as the factor of the difference between the experiment and simulation. Except for the source size, they could not explain the difference. In the case of the effective source size, assuming the brightness of our CFEG source to be the reported value of $1 \times 10^{9} \mathrm{~A} /\left(\mathrm{cm}^{2} \mathrm{~s} \mathrm{rad}\right)$ [8], Gaussian probe size, $\mathrm{S}$, was obtained to be $31 \mathrm{pm}$ at an electron probe current of $\mathrm{I}=30 \mathrm{pA}$ and convergent beam angle of $\alpha=30 \mathrm{mrad}$ by

$$
S^{2}=4 \ln 2 \frac{I}{B \pi^{2} \alpha^{2}}
$$

The estimated Gaussian probe size of $31 \mathrm{pm}$ is smaller than Gaussian convolution size of $70 \mathrm{pm}$. This result, however, is obtained in the case of coherent. In the case of partial coherent, the estimated Gaussian probe is expected to be larger than $31 \mathrm{pm}$ and to be equal to $70 \mathrm{pm}$ [9]. Therefore, we consider that the factor of the difference between the experiment and simulation may be attributed to be the partial coherence of electron waves at the electron source.

\section{References}

[1] K. V. Benthema, et al., Appl. Rev. Lett. 87 (2005) 034104.

[2]Y. Oshima, et al., Phys. Rev. B 81 (2010) 035317.

[3] S. Kim, et al., Appl. Phys. Exp. 3 (2010) 081301.

[4] J. M. LeBeau, et al., Phys. Rev. Lett. 100 (2008) 206101.

[5] J. M. LeBeau, et al., Phys. Rev. B 79 (2009) 214110.

[6] J. M. LeBeau, et al., Phys. Rev. B 80 (2009) 174106.

[7] S. Kim, et al., J. Elec. Micro. 60 (2011) dfq084.

[8] H. Sawada et al., J. Elec. Micro. 58 (2009) 357.

[9] This work was supported by the Japan Science and Technology Agency (JST) under the CREST project.

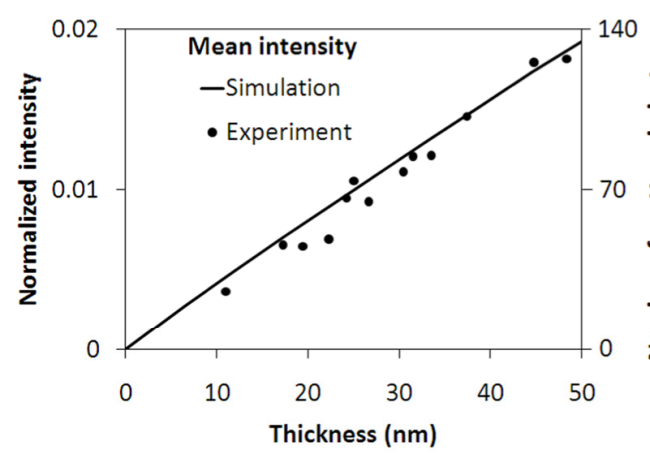

Fig. 1 Mean intensity as a function of the specimen thickness.

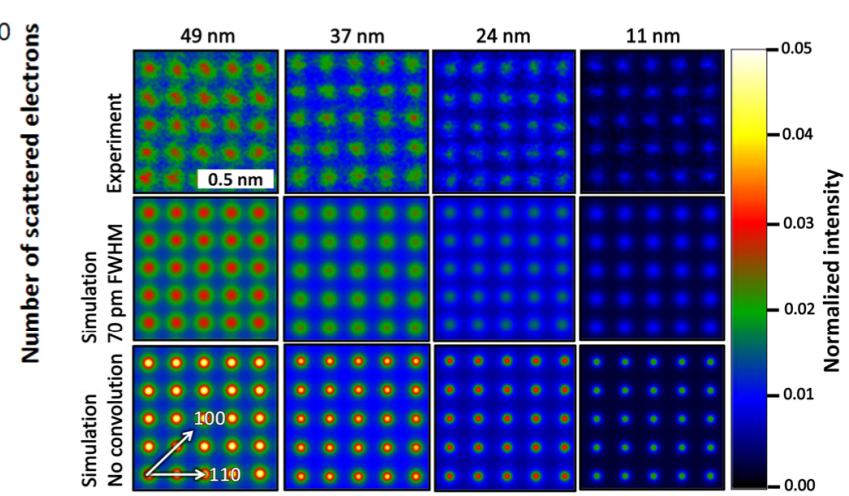

Fig. 2 Typical ADF images at thicknesses of 49, 37, 24 and $11 \mathrm{~nm}$. (first row) the experimental images, (second) the simulated images convoluted by a Gaussian function. (third) the simulated images. 